Article

\title{
Graphene prepared via the dry ice in flames method and its pu- rification using different routes: a comparative study
}

\author{
Eduardo Cuadros-Lugo ${ }^{1}$, Harby A. Martinez-Rodríguez ${ }^{1,2}$, Daniel Lardizabal-Gutierrez ${ }^{1}$, Ivanovich Estrada-Guel ${ }^{1}$, \\ Jose M. Herrera-Ramirez ${ }^{1, *}$ and Caleb Carreño-Gallardo ${ }^{1, *}$
}

1 Centro de Investigación en Materiales Avanzados, CIMAV, Miguel de Cervantes 120, Chihuahua, Chih 31136, Mexico; eduardo.cuadros@cimav.edu.mx (E.C.L.); harby.martinez@cimav.edu.mx (H.A.M.R.); daniel.lardizabal@cimav.edu.mx (D.L.G.); ivanovich.estrada@cimav.edu.mx (I.E.G.); martin.herrera@cimav.edu.mx (J.M.H.R.); caleb.carreno@cimav.edu.mx (C.C.G.)

2 Grupo de propiedades térmicas dieléctricas de compósitos, Universidad Nacional de Colombia, sede Manizales, Colombia

* Correspondence: martin.herrera@cimav.edu.mx, Tel.: +52 614 439-48-27; caleb.carreno@cimav.edu.mx, Tel.: +52 614 439-48-16

\begin{abstract}
Although the dry ice method used to synthesize exfoliated graphite/graphene is little known and used, it has significant advantages over others: it is low cost, simple, and a large quantity of material can be obtained using some inorganic and highly available acids (which can be reused). Despite the above advantages, the main reason for its incipient development is the resulting presence of magnesium oxide in the final product. In the present work, three different treatments were tested to remove this remnant using some acid chemical leaching processes. Based on the experimental evidence, it was found that using aqua regia, among other chemical mixtures, was the most efficient way of removing such as remnant, the residue being only $0.9 \mathrm{wt}$. $\%$. A mandatory highenergy mechanical milling stage was necessary during this treatment, in order to expose and dissolve the highly insoluble oxide without secondary chemical reactions on the graphenes. The obtained surface area was $504 \mathrm{~m}^{2} \mathrm{~g}^{-1}$; this high value resulted from the intense exfoliation can potentiate the use of this material for a wide variety of applications.
\end{abstract}

Keywords: graphene; dry ice; exfoliated graphite; magnesium oxide

\section{Introduction}

Although 16 years have passed since its discovery, graphene is still of great interest to the scientific community [1]. Its applications cover multiple fields such as sensors, composite strengthening [2], hydrogen storage, and lithium ion batteries, among others. The number of articles written on these topics since 2013 is around 15000 per year. Today, several synthesis methods are well known, being some of them the Hummer route [3], laser ablation, CVD [4], and high-energy milling [5]. Most of these methods involve serious disadvantages such as environmental pollution concerns [6] due mainly to the use of toxic reagents like sulfuric acid [7], potent reducing agents like hydrazine [8], or hazardous aromatic solvents like toluene, benzene, etc. [9]. Some others need expensive equipment for their production or require the use of high-purity gases [10]. The synthesis method using carbon dioxide as a precursor, which is known as dry ice in flames method, was developed by Chakrabarti and co-workers [11]. The method consists of igniting magnesium metal in a carbon dioxide $\left(\mathrm{CO}_{2}\right)$ atmosphere using a block of dry ice. This results in a highly exothermic reaction, reaching a temperature above $2500^{\circ} \mathrm{C}$, enough to perform the following chemical reaction [12]:

$$
2 \mathrm{Mg}(\mathrm{s})+\mathrm{CO}_{2}(\mathrm{~g}) \rightarrow 2 \mathrm{MgO}(\mathrm{s})+\mathrm{C}(\mathrm{s})
$$

The obtained products are magnesium oxide $(\mathrm{MgO})$ and carbon in graphite form. The latter can be presented in different forms, from exfoliated graphite to graphene. While exfoliated graphite can have large clusters of hundreds of graphene layers, graphene is 
made up of only a few sheets $(\sim 10)$. The main disadvantage of this method lies with the product separation: magnesium oxide from exfoliated graphite and graphenes. The predominant purification method reported in different studies consists of a chemical dissolution with hydrochloric acid $(\mathrm{HCl})$, forming magnesium chloride $\left(\mathrm{MgCl}_{2}\right)$, which is soluble in water, and its elimination by washing with demineralized water until a neutral $\mathrm{pH}$ is reached, then the purified exfoliated graphite is dried [13, 14]. Unfortunately, there is a problem related to a considerable amount of oxide (up to 5 at. \%), which is impossible to remove, this remnant contamination being undesirable for some applications. Even though this production method has numerous advantages such as low production costs, in addition to the fact that the amount of obtained product is far above the reached through other synthesis techniques used [15]. Even when the process uses acid for purification, the washing waters are quickly neutralized, avoiding their corrosive nature. For all these reasons, some authors consider it an eco-friendly method.

The present study proposes different chemical purification routes of the dry ice in flames products because magnesium oxide is not easy to dissolve fully. For this purpose, three acid solutions were tested, combining the leaching process with high-energy ball milling at reduced processing times. To follow the changes and processing differences, some samples of the exfoliated graphite/graphene were analyzed before and after the purification process through different characterization techniques, such as Raman spectroscopy, surface area analysis (BET), transmission electron microscopy (TEM) and thermogravimetric analysis (TGA).

\section{Materials and Methods}

\subsection{Synthesis Method}

The exfoliated graphene-graphite synthesis was carried out using a 20-cm solid cubic block of dry ice (solid carbon dioxide), to which a cavity of $10 \mathrm{~cm}$ in deep and $7 \mathrm{~cm}$ in diameter was made. $20 \mathrm{~g}$ of pure magnesium chips (Sigma-Aldrich, 6-35 mesh, 99.98\% purity) were introduced in the cavity and the reaction was induced through a spark provided by a butane gas lighter (Figure 1). This operation was repeated until a total of $150 \mathrm{~g}$ was obtained; as mentioned, the obtained material consisted of a mixture of graphite/graphene and magnesium oxide in powder form.
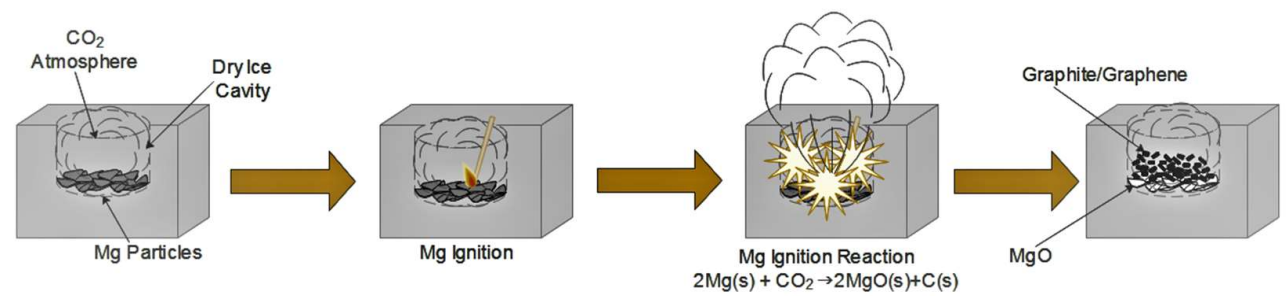

Figure 1. Descriptive diagram of the synthesis route to obtain exfoliated graphite/graphene.

\subsection{Purification Methods}

The obtained powders were subjected to a chemical purification process based on three different acid solutions (Table 1).

Table 1. Acid leaching solutions used during the purification processing.

Name Composition Nomenclature

Hydrochloric acid

$\mathrm{HCl} 1 \mathrm{M}$

LM1 


\begin{tabular}{cll} 
Aqua regia & $\mathrm{HNO}_{3}: \mathrm{HCl} 3: 1$ (in vol.) & LM2 \\
\hline Piranha solution & $\mathrm{H}_{2} \mathrm{SO}_{4}: \mathrm{H}_{2} \mathrm{O}_{2}(30$ vol. $\%)$ 3:1 & LM3
\end{tabular}

A general scheme of the involved processes used to remove magnesium oxide is presented in Figure 2. Two purification processes were performed in the present research. The first process (PP1) consisted of leaching the graphite/graphene synthesis product with the three solutions indicated in Table 1, followed by vacuum filtering. In the second process (PP2), the material obtained from PP1 was further processed by mechanical milling. Then it was leached again with the same acid solution of the first leaching process and vacuum filtered. The obtained samples were characterized through the techniques mentioned above. The main reason to complement the leaching with high-energy ball milling is that the mechanical milling causes the removal of the carbon layers that are firmly attached to the $\mathrm{MgO}$ and, as a consequence, it promotes an increased chemical attack which enhances the dissolution of these unwanted particles.



Figure 2. Illustrative diagram of sample processing.

\subsection{Leaching}

${ }^{\circ} \mathrm{C}$.

The leaching process was set to $24 \mathrm{~h}$ for all samples under a constant stirring at 80

\subsection{Vacuum filtering}

After the leaching process, a vacuum filtration process was carried out in the wet mixtures using a 500-mL Kitasato flask coupled with a Büchner funnel and a Whatman filter paper number 42 . The powders were washed with demineralized water until reaching neutral $\mathrm{pH}$ and dried in a laboratory stove at $100^{\circ} \mathrm{C}$ overnight.

\subsection{Milling}

High-energy milling was carried out using a Spex $8000 \mathrm{M}$ device, processing $1 \mathrm{~g}$ of the washed and dried sample with a milling time of $30 \mathrm{~min}$. A hardened steel vial and 13$\mathrm{mm}$ balls were used; the ball-to-powder weight ratio was kept at 30:1 (in weight) for all experimental runs and no process control agent was added during the milling. 


\subsection{Characterization techniques}

The presence of phases and their crystalline characteristics were studied by X-ray diffraction (XRD) using a Panalytical X'Pert-Pro diffractometer working at $40 \mathrm{kV}$ and 35 $\mathrm{mA}$ using $\mathrm{Cu}-\mathrm{K} \alpha$ radiation with a wavelength of $0.154056 \mathrm{~nm}$; the data were collected in the $2 \theta$ range from 5 to $80^{\circ}$ with a step size of $0.2^{\circ}$ per second. Thermogravimetric analysis (TGA) was performed using a TA Instrument model Q600, with a heating ramp of 10 ${ }^{\circ} \mathrm{C} / \mathrm{min}$ from room temperature to $800{ }^{\circ} \mathrm{C}$ under an air flow of $50 \mathrm{~cm}^{3} / \mathrm{min}$. Raman spectrometry was performed using a LabRam HR VIS-633 microscope developed by Horiba equipped with a He-Ne laser source. Transmission electron microscopy (TEM) using a Hitachi 7700 microscope and high-resolution transmission electron microscopy (HRTEM) using a TEM JEOL JEM 2200FS microscope were employed to analyze the synthesized graphenes. The surface area was determined by the Brunauer-Emmett-Teller (BET) method in a Quanta chrome model Nova 4200e analyzer, taking 11 points of 0.05 to 0.3 of relative pressure $\left(\mathrm{P} / \mathrm{P}_{0}\right)$.

\section{Results}

\subsection{Characterization of graphene powders}

TEM studies were carried out to determine the phase coexistence, particle size, morphologies and microstructure of samples. The obtained material from the dry ice synthesis process without any additional processing was analyzed. Figure 3 shows a TEM micrograph where a mixture of graphite/graphene structures is observed, as well as the presence of a considerable concentration of $\mathrm{MgO}$ in the form of cubical particles. The proposed purification processing methods are intended to remove these agglomerated and exposed particles.

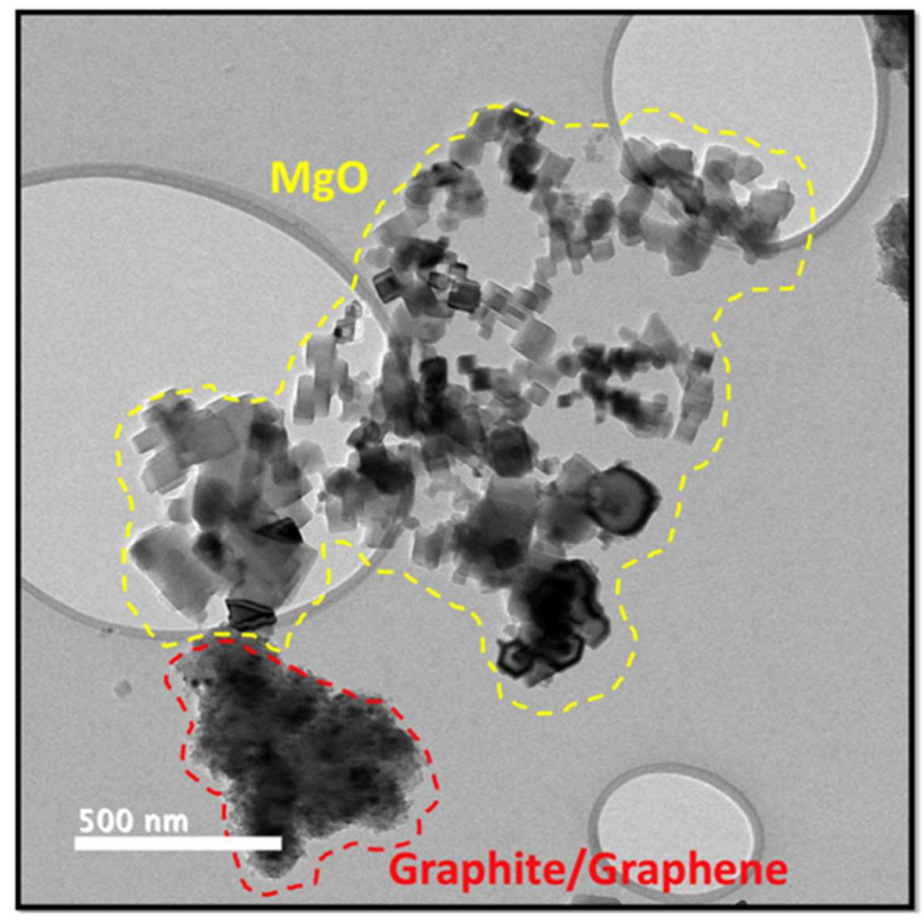

Figure 3. TEM image of raw sample obtained after the synthesis.

Figure 4 shows HRTEM micrographs of samples after the purification processes. Micrographs in Figures 4a-c correspond to samples processed by the first purification 
process (PP1), where the presence of graphene sheets and embedded $\mathrm{MgO}$ nanoparticles is clear. These nanoparticles have the characteristic cubic geometry, whose size dispersion varies from 10 to $20 \mathrm{~nm}$. Micrographs in Figures $4 \mathrm{~d}$-f correspond to samples subjected to the first purification process (PP1) followed by mechanical milling and a second purification process (PP2) to remove as much as possible the $\mathrm{MgO}$ phase. These images show clear evidence of a graphene sheet (Figure $4 \mathrm{~d}$ ), where the van der Waals interlayer attractions allowed the nanosheets to slide each other perpendicularly to the caxis. Still, there is enough attraction preventing the complete formation of individual graphene monolayers, where the diffraction contrast is related to thickness variation, denoting the presence of multiple graphene layers and polycrystalline structures with randomly oriented grains. After the second leaching process, the relative concentration of $\mathrm{MgO}$ particles decreased considerably compared to samples from the first leaching process.
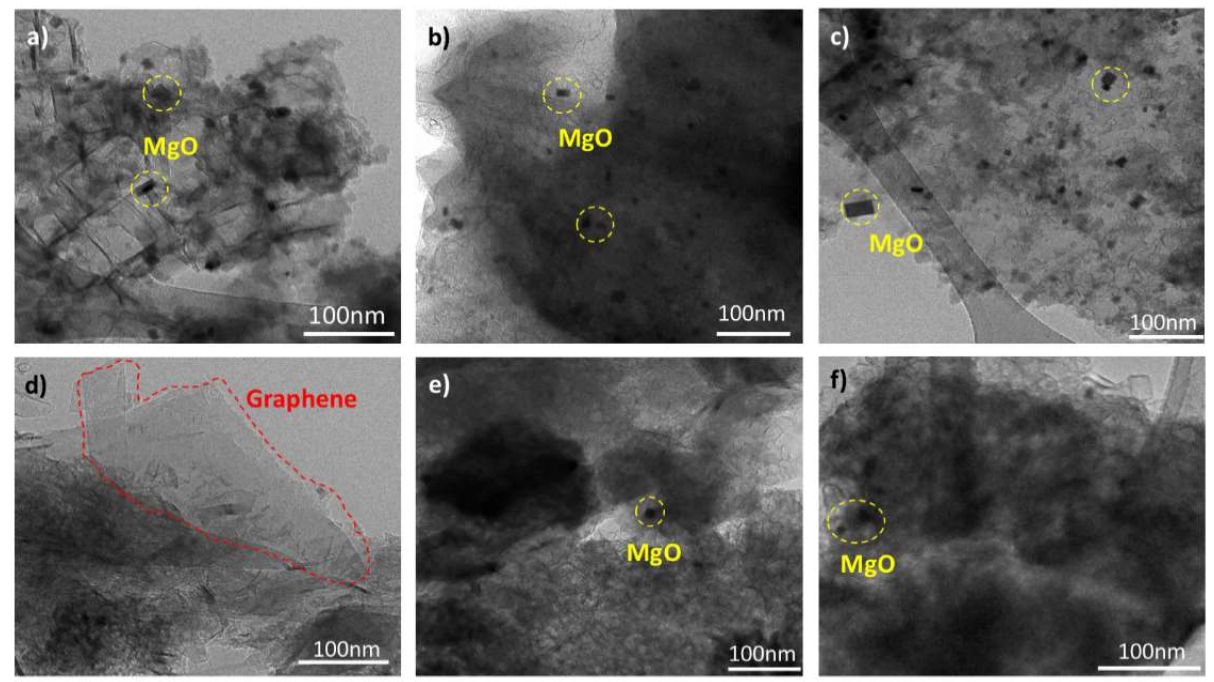

Figure 4. HRTEM images of a) LM1-PP1, b) LM2-PP1, c) LM3-PP1, d) LM1-PP2, e) LM2-PP2 and f) LM3-PP2 samples.

Comparative X-ray diffraction patterns of samples under different processing conditions are seen in Figure 5. Figure 5a presents the diffractograms of samples after the first purification process. The indexed diffraction peaks located at $2 \theta \approx 26$ and $54^{\circ}$ are attributed to the planes (002) and (004), respectively, both corresponding to exfoliated graphite (JCPDS 41-1487). The signals at 37, 43, 63, 75 and $78^{\circ}$ are correlated with the planes (111), (200), (220), (311), and (222), respectively, and are attributed to magnesium oxide (JCPDS 78-0430). Figure 5b presents the diffractograms of samples after the second purification process, where a great decrease in the $\mathrm{MgO}$ signals is evidenced, although it is still present. Due to the instrumental restriction of $\mathrm{XRD}$, this analysis does not allow us to differentiate which purification method is better quantitatively. 

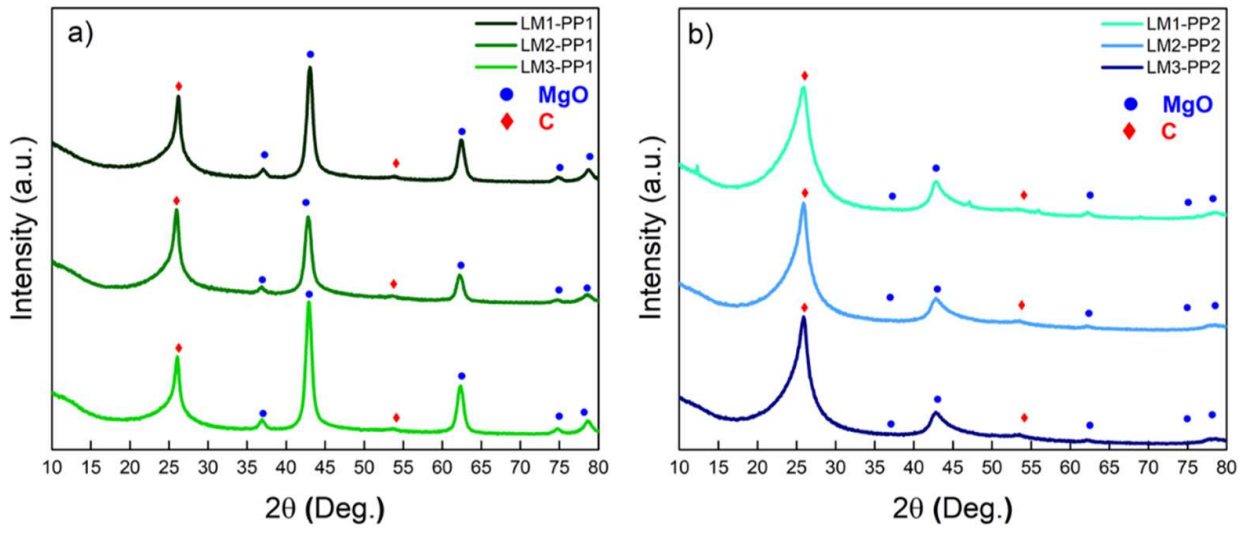

Figure 5. XRD patterns of a) PP1 and b) PP2 samples.

The number of layers along the c-axis $\left(\mathrm{N}_{\mathrm{c}}\right)$ of graphene samples was calculated by the equation described by Seehra et al. [16] (Equation 1), where $L_{c}$ is the apparent crystallite size, which was determined from the Scherrer equation (Equation 2) and doo2 is the interplanar spacing of for the (002) plane, which was calculated from the Bragg's Law (Equation 3) [17, 18].

$N c=\frac{L_{c}}{d_{002}}$
$L_{c}=\frac{k \lambda}{\beta \cos \theta}$
$d_{002}=\frac{n \lambda}{2 \sin }$

As can be seen in Table 2, the number of layers decreases after going through the purification process 2 .

Table 2. Numbers of layers in graphene samples.

\begin{tabular}{cc}
\hline Sample & Number of layers \\
\hline LM1-PP1 & 32 \\
\hline LM2- PP1 & 26 \\
\hline LM3- PP1 & 31 \\
\hline LM1- PP2 & 23 \\
\hline LM2- PP2 & 13 \\
\hline LM3- PP2 & 26 \\
\hline
\end{tabular}

Figure 6 displays the results of Raman spectroscopy. The Raman spectra evidence that all samples are basically composed of a carbonaceous matrix with representative signals related to graphitic structures; these signals are commonly described as D, G, and 2D bands, which were detected at 1350,1580, and $2600 \mathrm{~cm}^{-1}$, respectively [19]. As it is well known, the $\mathrm{D}$ band is related to lattice disorder and sp3 defects in graphenes, while the $G$ band is the result of in-plane C-C symmetric stretching vibrations and is associated with the sp2 structure of carbon [20]. The 2D band is correlated with the overtone of the $\mathrm{D}$ band [21]. The D-band and G-band (ID/IG) intensity ratio is used to evaluate the degree of 
disorder and defects in the graphitic structure. As can be observed, the ID/IG ratio was affected by the mechanical milling process increasing from 0.91 (average of the three leaching procedures) from the first purification process (PP1) to 1.24 for the second purification process (PP2), which involves mechanical milling. A minimal decrease in the $2 \mathrm{D}$ band can also be seen, which can be attributed to the onset of defects in the graphene structure [22].

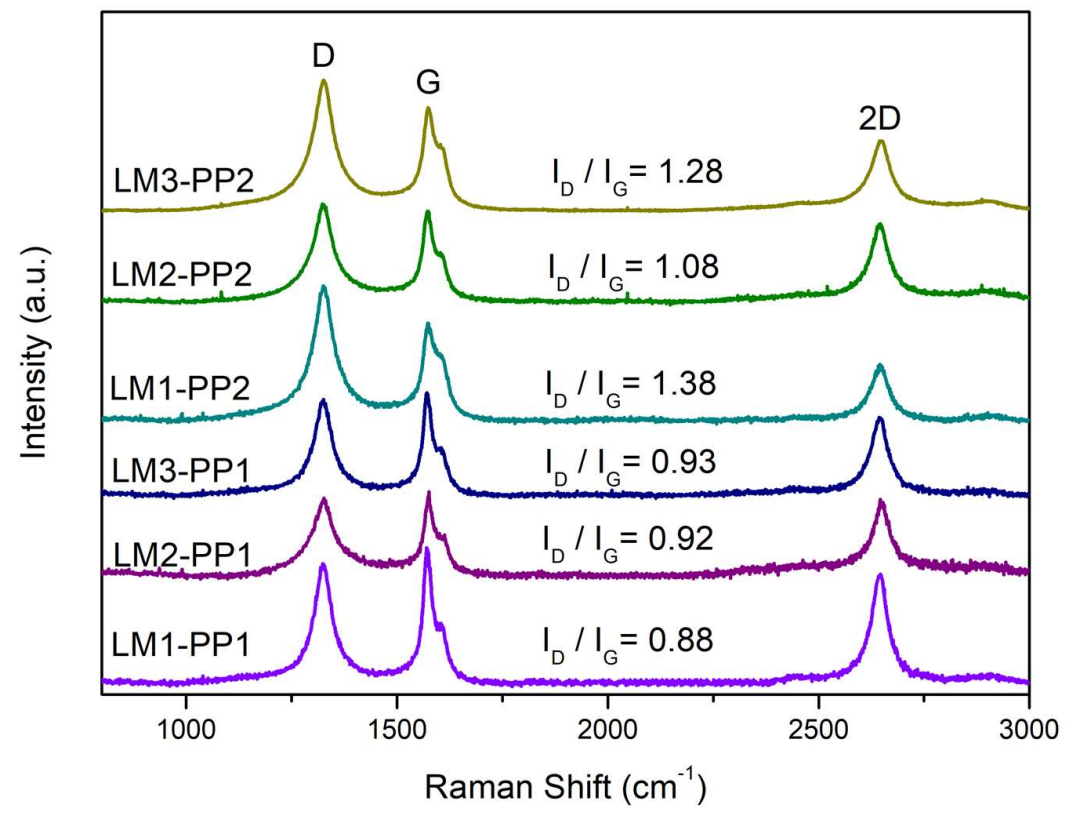

Figure 6. Comparative Raman spectra of PP1 and PP2 leached samples.

Table 3 presents the results of the surface area (SA) analysis attained through the BET method. The values for PP1 samples are in the range of $300-390 \mathrm{~m}^{2} \mathrm{~g}^{-1}$, while the PP2 samples presented higher and uniform values in the range of $502-504 \mathrm{~m}^{2} \mathrm{~g}^{-1}$. With this, it can be pointed out that SA is a dependent variable closely related to the purification treatment.

Table 3. Summary chart of SA (in $\left.\mathrm{m}^{2} \mathrm{~g}^{-1}\right)$ of samples after leaching.

\begin{tabular}{cc}
\hline Sample & Surface Area \\
\hline LM1-PP1 & $332.6 \pm 1$ \\
\hline LM2- PP1 & $389.0 \pm 1$ \\
\hline LM3- PP1 & $312.6 \pm 1$ \\
\hline LM1- PP2 & $504.8 \pm 1$ \\
\hline LM2- PP2 & $503.4 \pm 1$ \\
\hline LM3- PP2 & $502.0 \pm 1$ \\
\hline
\end{tabular}

The thermogravimetric analysis (TGA) curves show the mass loss of samples as a function of the temperature (Figure 7). The mass loss related to carbon oxidation occurs in the range of 500 to $750{ }^{\circ} \mathrm{C}$, leaving a solid residue mainly composed of magnesium oxide. The final residue percentage of PP1 samples is between 22.8 and $29.6 \%$, which is correlated as $\mathrm{MgO}$ (Figure 7a). This large amount of residue is the main reason why this 
synthesis method has not been widely developed. For PP2 samples (Figure 7b), the final residue percentage ranges from 1.0 to $2.9 \%$, standing out the leaching process where aqua regia is used (LM2) with a residue of only $1.0 \%$. TGA analysis is a fundamental tool for evaluating the best purification treatment (related to residue generation after calcination) on a quantitative basis.
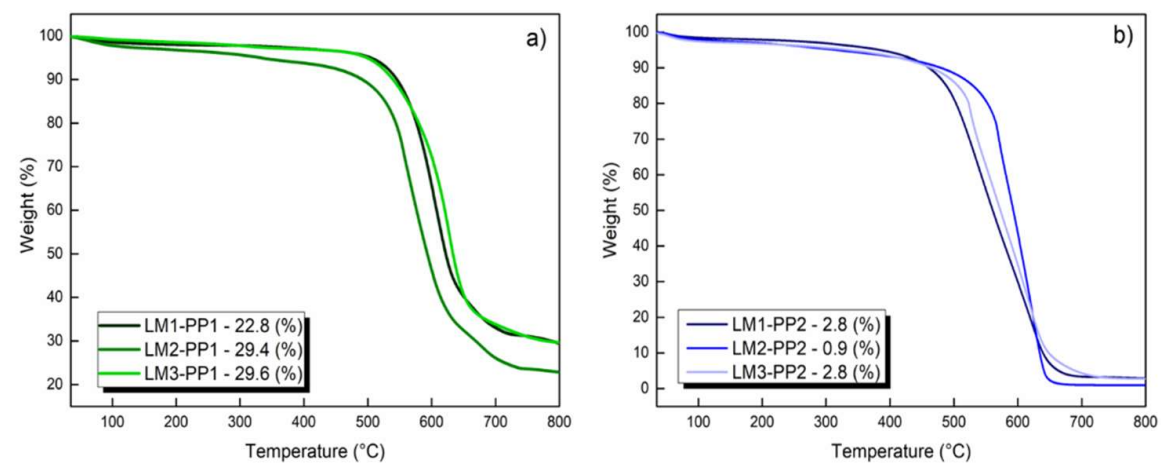

Figure 7. Comparative thermograms and residues of leached samples a) PP1 and b) PP2.

\section{Discussion}

Based on the evidence obtained by TEM, a mechanism for the high concentration of unreacted $\mathrm{MgO}$ during the dry ice in flames synthesis method is proposed, which is schematized in Figure 8. Figure 8a shows a general view of the $\mathrm{MgO}$ nanoparticles surrounded by graphite. The TEM image in Figure $8 \mathrm{~b}$ evidences that the $\mathrm{MgO}$ nanoparticles are covered with several graphene layers, preventing physical contact with the leaching chemical from achieving their complete dissolution. These layers may be present in different thicknesses spanning few layers; if the material reaches many layers, it can induce the graphite formation.

It is worth mentioning that van der Waals forces play an important role, as they are responsible for linking these graphene sheets together during the graphite formation. Based on the surface area results, it can be assumed that most of the particles remain as exfoliated graphite consisting of a few hundred graphene layers. Note that the theoretical surface area value for graphene is $2700 \mathrm{~m}^{2} \mathrm{~g}^{-1}$ [23], which is well above the values found for the materials synthesized in this investigation; this may indicate how far we are from generating a graphene monolayer. The mechanical milling process promotes the graphite exfoliation and induces an increase of surface exposure of the oxide; this facilitates the acid attack and the subsequent dissolution process (Figure 8c).

Another clear advantage of this purification process is related to the processing time to obtain a relatively elevated surface area $\left(\sim 500 \mathrm{~m}^{2} \mathrm{~g}^{-1}\right)$. Indeed, such a value of the surface area is achieved after $8 \mathrm{~h}$ when using high-energy ball milling [24], while the time used to carry out the exfoliation in this work was only $30 \mathrm{~min}$, which is reflected in a considerable saving of time, in addition to being able to process a greater quantity of material. Furthermore, our SA value is almost twice compared to methods of synthesis like the reduction of graphene oxide, where a surface area of $298.2 \mathrm{~m}^{2} \mathrm{~g}^{-1}$ was obtained [25]. 


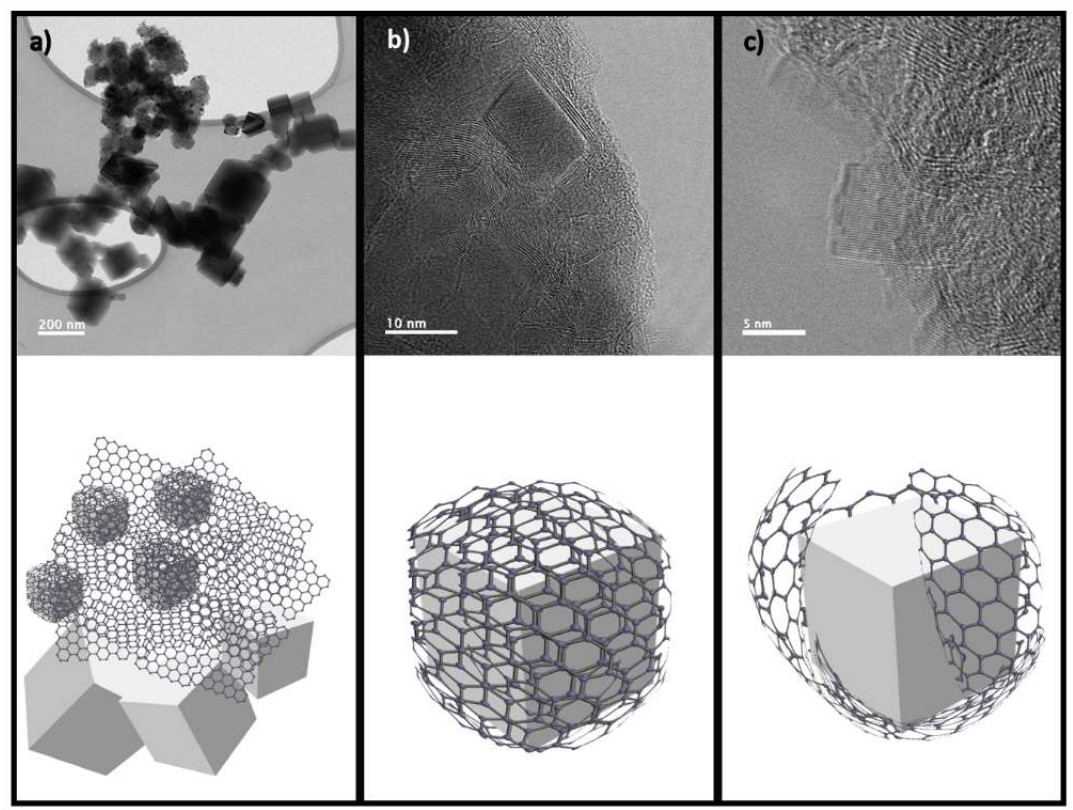

Figure 8. TEM micrographs and proposed dissolution mechanism.

\section{Conclusions}

The dry ice in flames method was successfully used for exfoliating graphite/graphene. This method can be a potential alternative to perform this process due to its low cost, simplicity and high scalability. The proposed mechanical milling stage and a second leaching process (PP2) are essential for the effective removal of $\mathrm{MgO}$. Based on the experimental results, the treatment with the use of aqua regia in the PP2 process (LM2-PP2) was the most effective, reaching a value of $1 \%$ of $\mathrm{MgO}$ residues. This route is more effective than that of the treatment with hydrochloric acid (LM1), which is universally used on this subject. The study opens the doors for using the purified exfoliated graphite for various applications where a high surface area is necessary, such as in catalysis and removal of solvents and heavy metals.

Author Contributions: Conceptualization, C.C.G. and J.M.H.R.; methodology, C.C.G., I.E.G., D.L.G; formal analysis, E.C.L., H.A.M.R., I.E.G., D.L.G., C.C.G. and J.M.H.R.; investigation, E.C.L., H.A.M.R., I.E.G., D.L.G., C.C.G. and J.M.H.R.; writing-original draft preparation, C.C.G. and J.M.H.R.; writing - review and editing, I.E.G., D.L.G., C.C.G. and J.M.H.R; visualization, E.C.L. and H.A.M.R.; supervision, I.E.G., C.C.G. and J.M.H.R.; project administration, C.C.G. and J.M.H.R. All authors have read and agreed to the published version of the manuscript.

Funding: This research received no external funding.

Acknowledgments: The authors would like to thank the valuable technical assistance of R. Ochoa Gamboa, P. Pizá Ruiz, L. de la Torre Saenz, J.E. Ledezma Sillas and E. Guerrero Lestarjette.

Conflicts of Interest: The authors declare that they have no known competing financial interest or personal relationships that could have influenced the work reported in this paper.

\section{References}

1. Novoselov, K.S., et al., A roadmap for graphene. Nature, 2012. 490(7419): p. 192-200. 
2. Rashad, M., et al., Effect of Graphene Nanoplatelets addition on mechanical properties of pure aluminum using a semi-powder method. Progress in Natural Science: Materials International, 2014. 24(2): p. 101-108.

3. Paulchamy, B., G. Arthi, and L. Bd, A Simple Approach to Stepwise Synthesis of Graphene OxideNanomaterial. Journal of Nanomedicine \& Nanotechnology, 2015.6 (1): p. 1000253.

4. Castro Beltrán, A., et al., Obtención de grafeno mediante la reducción química del óxido de grafito. Ingenierías, 2011. 14(52): p. 3442.

5. Kim, D., et al., Synthesis of reduced graphene oxidelaluminum nanocomposites via chemical-mechanical processes. Journal of Composite Materials, 2018. 52(22): p. 3015-3025.

6. Luo, D., et al., An improved method to synthesize nanoscale graphene oxide using much less acid. Materials Today Physics, 2019. 9: p. 100097.

7. Papageorgiou, D.G., I.A. Kinloch, and R.J. Young, Mechanical properties of graphene and graphene-based nanocomposites. Progress in Materials Science, 2017. 90: p. 75-127.

8. Kairi, M.I., et al., Toward high production of graphene flakes - a review on recent developments in their synthesis methods and scalability. Journal of Materials Chemistry A, 2018. 6(31): p. 15010-15026.

9. Jeon, I.Y., et al., Edge-carboxylated graphene nanosheets via ball milling. Proceedings of National Academic of Sciences U S A, 2012. 109(15): p. 5588-93.

10. García Martínez, V., Estudio de la estabilidad del óxido de grafeno con el tiempo. 2013, Universidad de Oviedo.

11. Chakrabarti, A., et al., Conversion of carbon dioxide to few-layer graphene. Journal of Materials Chemistry, 2011. 21(26): p. 94919493.

12. Zhang, J., et al., Synthesis of graphene from dry ice in flames and its application in supercapacitors. Chemical Physics Letters, 2014. 591: p. 78-81.

13. Shams, S.S., R. Zhang, and J. Zhu, Graphene synthesis: a Review. Materials Science-Poland, 2015. 33(3): p. 566-578.

14. Arifutzzaman, A., et al., Fabrication and Characterization of Graphene from Solid Carbon Dioxide. Advanced Materials Research, 2015. 1115: p. 418-421.

15. Kim, T.H., et al., Bulk synthesis of graphene-like materials possessing turbostratic graphite and graphene nanodomains via combustion of magnesium in carbon dioxide. Carbon, 2019. 149: p. 582-586.

16. Albetran, H.M., Structural Characterization of Graphite Nanoplatelets Synthesized from Graphite Flakes. Preprints, 2020. 2020080325.

17. Low, I.-M., H.M. Albetran, and M. Degiorgio, Structural characterization of commercial graphite and graphene materials. Journal of Nanotechnology and Nanomaterials, 2020. 1(1): p. 23-30.

18. Low, I.M., et al., A comparative study on crystallization behavior, phase stability, and binding energy in pure and Cr-doped TiO2 nanotubes. Journal of Materials Research, 2013. 28(3): p. 304-312.

19. Al-Sherbini, A.-S., et al., Exfoliation of graphene sheets via high energy wet milling of graphite in 2-ethylhexanol and kerosene. Journal of Advanced Research, 2017. 8(3): p. 209-215.

20. Zhou, W., et al., Creation of individual few-layer graphene incorporated in an aluminum matrix. Composites Part A: Applied Science and Manufacturing, 2018. 112: p. 168-177.

21. Zan, Y.N., et al., Introducing graphene (reduced graphene oxide) into Al matrix composites for enhanced high-temperature strength. Composites Part B: Engineering, 2020. 195: p. 108095.

22. Lin, F., et al., Synergistic effects of TiC and graphene on the microstructure and tribological properties of Al2024 matrix composites. Advanced Powder Technology, 2021. 32(10): p. 3635-3649.

23. Priyanka, V., et al., Physicochemical exfoliation of graphene sheets using graphitic carbon nitride. New Journal of Chemistry, 2019. 43(41): p. 16200-16206. 
24. Mahmoud, A.E.D., A. Stolle, and M. Stelter, Sustainable Synthesis of High-Surface-Area Graphite Oxide via Dry Ball Milling. ACS Sustainable Chemistry \& Engineering, 2018. 6(5): p. 6358-6369.

25. Tiwari, J.N., et al., Reduced graphene oxide-based hydrogels for the efficient capture of dye pollutants from aqueous solutions. Carbon, 2013. 56: p. 173-182. 